Applied Mathematical Sciences, Vol. 7, 2013, no. 54, 2687 - 2707

HIKARI Ltd, www.m-hikari.com

\title{
Mathematical Analysis of a Comprehensive HIV AIDS Model: Treatment versus Vaccination
}

\author{
M. O. Okongo \\ Department of Physical Sciences \\ Chuka University, Box +254 60400 109, Chuka, Kenya \\ markokongo@yahoo.com
}

J. Kirimi

Department of Physical Sciences

Chuka University, Box 109, Chuka, Kenya

h.kirimi@yahoo.com

\author{
A. L. Murwayi \\ Department of Physical Sciences \\ Chuka University, Box 109, Chuka, Kenya \\ almurwayi@yahoo.com \\ D. K. Muriithi \\ Department of Physical Sciences \\ Chuka University, Box 109, Chuka, Kenya \\ kamuriithi@yahoo.com
}

Copyright (c) 2013 M. O. Okongo et al. This is an open access article distributed under the Creative Commons Attribution License, which permits unrestricted use, distribution, and reproduction in any medium, provided the original work is properly cited.

\begin{abstract}
A comprehensive deterministic HIV/AIDS transmission model incorporating social behaviour, treatment, vaccination, stages of infection, age structures, discrete time delay and vertical transmission is presented and rigorously analyzed. Two age structures are considered with group one consisting of children aged (0 - a) years and group two consisting of adults aged (a) years and above. In this study we investigate wether
\end{abstract}


a trade-off exists between vaccination and treatment. Numerical simulations shows that treatment that does not reduce infectiousness is worse than when the treatment is not applied at all, however when coupled with effective counseling, then it is very effective in combating the spread of the disease and finally eliminating it. A trade off seems to exists between vaccination and treatment and therefore careful considerations should be made when vaccination and treatment is to be applied together because a combination of the two could be counterproductive or helpful depending on how it is implemented..

Keywords: Equilibrium, Stability, The Basic Reproduction Number $-R_{0}$, The Next Generation Matrix

\section{Introduction}

Mathematical models and Computer simulations are useful experimental tools for building and testing theories, assessing quantitative conjectures, determining sensitivities to changes in parameter values and answering specific questions.

The magnitude and severity of the problem of HIV/AIDS became increasingly evident in the early 1990's with a prevalence rate of about 12 percent in Africa [38]. In some communities i.e in Uganda a whole generation was wiped out [34].

The first simple HIV Mathematical epidemic model goes back to Anderson [26] in 1986. By then behaviour change was recognized as the major way of combating the spread of HIV/AIDS epidemic given that there was no treatment or vaccine to the virus.

After the discovery of Anti-retroviral treatment, modeling of HIV/AIDS was directed towards incorporating behaviour change and effects of treatment. Incorporating treatment and social behaviour posed a challenge to HIV/AIDS mathematical modeling because treatment acts both in the positive and negative direction. It reduces the infectiousness of an infected individual reducing the probability of transmission from an infective to a susceptible. On the contrary anti- retroviral therapies increases the lifespan of the HIV Infectives and as such they can infect more people if the treatment does not reduce infectiousness with no change in social behaviour.

These directions included the models by Valesco - Hernandez and Hsieh 1994 [16] who concluded that only significant reductions in the transmission probability can contain the spread of the epidemic. Such reductions could be through adoption of safer sexual practices or through reductions in viral load due to treatment. A model by Ying -Yen and Cooke [17] in 2000 on behaviour change and treatment of core groups and its effects on the spread of 
HIV/AIDS showed that behaviour change and treatment can eradicate the disease however if the treatment and behaviour change levels do not reach critical values, detrimental effects could be realized resulting from slower progresion to AIDS without sufficiently lower transmission rates resulting in increased spread of HIV infection. Raw et al, [20] in 2001, modeled the effect of combination of anti-retroviral treatments with different levels of unsafe sex on HIV incidence in Australia when effective anti-retroviral treatments first became widely available in Australia. The results suggested that decreases in HIV incidences through large decreases in infectiousness as a result of combination anti-retroviral treatment could be counterbalanced by much more modest increases in the levels of unsafe sex.

The struggle for a safe and effective vaccine has been on going for over twenty years but still remains a difficult target, however Recent studies done in Thailand hinted that some vaccine for some strain of the virus could be on the way with an efficacy level of about 30 percent [5]. Wells A.W [4] 1998, in his paper, "even imperfect vaccines could be valuable", suggested that vaccines against the AIDS causing virus could save money, extend lives and prevent deaths even if these vaccines are only moderately effective in preventing or treating the infection. Vaccination reduces the number of susceptibles in the population whereas treatment increases the lifespan of the infectives which may lead to an increase in the rate of infection. A trade off seems to exists between vaccination and treatment and therefore a combination of the two could be counterproductive or helpful.

This study have developed a comprehensive deterministic HIV/AIDS transmission model incorporating counseling, treatment, vaccination, stages of infection, Age structures, discrete time delay and vertical transmission. It is our view that this study represents the very first practical realistic comprehensive model harmonizing the existing models into a single model that can be used to study most aspects of HIV and AIDS without presenting conflicting findings thus attempts to investigate the possibility of a trade off between vaccination and treatment.

\section{Preliminaries}

The population is divided into two age groups. Group I comprises of the sexually Immature children aged (0 - a) years and group II comprises of sexually mature and active adults aged (a) years and beyond. It is group II that is responsible for the spread of the epidemic through sexual activity and for the spread in children through infected mothers (Vertical Transmission). 


\subsection{Assumptions}

1. The transmission of HIV from an infective to a susceptible is through heterosexual mode and vertical transmission.

2. There is random mixing of individuals within the population.

3. AIDS cases has full blown symptoms and are easily noticeable and are not sexually interacted with and as such, they don't transmit the virus and do not give birth to new borns.

4. Individuals in group I comprise of sexually Immature children aged (0 a) years and therefore do not transmit the disease.

5. The removed class are sexually interacted with but are not infectious and are immuned.

6. Treatment is done in the adult group only.

7. The vaccine acts both as the "Leaky type" and the "All or Nothing type" of vaccine. The "Leaky type" of vaccine protects everybody vaccinated partially while the "All or Nothing" type protects a fraction of those vaccinated fully leaving the rest completely susceptible.

\subsection{Parameters and Notations Used in the Model}

\subsubsection{Notations for the variables used in the model}

$W(t)$ - denotes the number of susceptible children at time $t$.

$H(t)$ - The number of infected children at time $t$.

$U(t)$ - The number of AIDS Cases at time $t$ in group I.

$S(t)$ - The number of susceptible adults at time $t$.

$V(t)$ - The number of vaccinated adults at time $t$.

$R(t)$ - The number of removed adults covered by the vaccine at time $\mathrm{t}$.

$X(t)$ - The number of infective adults at time $t$.

$Z(t)$ - The number of infected adults who receive treatment at time $t$.

$A(t)$ - The number of full blown AIDS Cases in group II at time $t$.

$P(t)$ - The total population size at time $t$.

\subsubsection{The Parameters used in the model and how they are obtained}

$m$ - denotes the rate at which the HIV infected children progress to AIDS in group I obtained from the average incubation period of HIV in children.

$\mathrm{d}$ - The disease related death rate obtained from the number of years it takes one who has shown full blown AIDS symptoms to die of the disease. 
$\mu$ - Natural death rate referring to the number of deaths in a year per 1000 people.

$\alpha$ - The proportion of susceptible adults assumed to be vaccinated taken arbitrary.

$\delta$ - vaccine efficacy, assuming the "All or Nothing type of vaccine". It represents the proportion of the vaccinated completely covered by the vaccine.

$\epsilon$ - Proportion of the infectives receiving treatment.

$\eta$ - The rate at which the infectives who do not receive treatment progress to HIV/AIDS calculated from the average incubation period of HIV in adult infectives.

$\lambda$ - Rate at which those treated progress to AIDS.(both the normal and vaccinated infectives) calculated from the average incubation period of treated adult infectives.

$b$ - The per capita birth rate calculated as a ratio of the average live births per year to the general population size.

g - Natural child mortality rate calculated as a ratio of the average number of children below 15 years who die per year to the total population below 15 years.

v - Proportion of babies born with HIV from HIV infected mothers.

$b_{1}$ - Per capita birth rate of the adults calculated as a ratio of the average live births per year among the adults to the general population size of the adults excluding AIDS cases.

a - Years at which an individual becomes sexually mature, $\beta_{1}$ - is the per partnership transmission probability of a normal infective who is not treated.

$\beta_{2}$ - is the per partnership transmission probability of an infective who is treated and counseled.

$c_{1}$ - is the average number of new sexual partners acquired per unit time by those infected but not yet counseled and treated.

$c_{2}$ - is the average number of new sexual partners acquired per unit time by those treated and counseled.

$\theta$ - The proportion of the vaccinated partially covered by the vaccine assuming the "leaky type of vaccine".

\subsection{Age - Structures}

Group I consists of children of age (0 - a) years which has three compartments namely $W(t), H(t)$ and $U(t)$. The differential equations for group I takes the 
following form:-

$$
\begin{aligned}
\frac{d W(t)}{d t} & =b_{1} N(t)-g W(t)-b_{1} v(X(t)+Z(t))-e^{(-g a)} b_{1} N(t-a) \\
\frac{d H(t)}{d t} & =b_{1} v(X(t)+Z(t))-(g+m) H(t) \\
\frac{d U(t)}{d t} & =m H(t)-(d+g) U(t)
\end{aligned}
$$

Group II consists of adults aged (a) years and beyond. It has six compartments comprising of $S(t), V(t), R(t), X(t), Z(t)$ and $A(t)$. The differential equations takes the following form:-

$$
\begin{aligned}
\frac{d S(t)}{d t}= & e^{(-g a)} b_{1} N(t-a)-\left(\frac{\beta_{1} c_{1} S(t)(X(t))}{N(t)}+\frac{\beta_{2} c_{2} S(t)(Z(t))}{N(t)}\right) \\
& -(\mu+\alpha) S(t) \\
\frac{d V(t)}{d t}= & (\alpha-\delta) S(t)-\left((1-\theta)\left(\frac{\beta_{1} c_{1} V(t)(X(t))}{N(t)}+\frac{\beta_{2} c_{2} V(t)(Z(t))}{N(t)}\right)\right) \\
& -\mu V(t) \\
\frac{d R(t)}{d t}= & \delta S(t)-\mu R(t) \\
\frac{d X(t)}{d t}= & \left(\frac{\beta_{1} c_{1} S(t)(X(t))}{N(t)}+\frac{\beta_{2} c_{2} S(t)(Z(t))}{N(t)}\right) \\
& +\left((1-\theta)\left(\frac{\beta_{1} c_{1} V(t)(X(t))}{N(t)}+\frac{\beta_{2} c_{2} V(t)(Z(t))}{N(t)}\right)\right) \\
& -(\mu+\epsilon+\eta) X(t) \\
\frac{d Z(t)}{d t}= & \epsilon X(t)-(\mu+\lambda) Z(t) \\
\frac{d A(t)}{d t}= & \eta X(t)+\lambda Z(t)-(\mu+d) A(t)
\end{aligned}
$$

With:

$P(t)=N(t)+W(t)+H(t)+U(t)+A(t), \quad N(t)=S(t)+V(t)+R(t)+X(t)+Z(t)$

and $b_{1}=b \frac{P(t)}{N(t)}$

The population growth model is given by the following differential equation.

$\frac{d P(t)}{d t}=\left(b_{1}-\mu\right) N(t)-g(W(t)+U(t)+H(t))-\mu(A(t))-d(U(t)+A(t))$

With $W(0)=f_{1} P(0), H(0)=0, U(0)=0$

$\mathrm{P}(0)$ being the the total population at the start of the epidemic and $f_{1}$, is the 
fraction of children (a) years and below at the start of the epidemic where $0<f_{1}<1$.

$S(0)=\left(1-\phi_{0}\right) f_{2} P(0), X(0)=\phi_{0} f_{2} P(0), A(0)=0$, with $0<f_{2}<1$, being the fraction of the population that were adults at the start of the epidermic. $\phi_{0}$, is the fraction of the adult population that were HIV infective at the start of the epidemic with $f_{1}+f_{2}=1$.

\section{Analysis of the Disease Free Equilibrium (D.F.E)}

We analyze the stability of the Disease Free Equilibrium, (D.F.E) in group II since it is this group that is sexually active and responsible for the spread. We also assume that the AIDS cases $A(t)$ in the population can easily be identified from the full blown symptoms and are not associated with sexually and as such are not involved in the spread of the diseases though their projection will be investigated numerically to identify the impact they have on the population.

\subsection{Proportional Variables}

We use the proportions of the populations to enable us study the steady states. This is based on the assumption that it is more likely for the population proportions to attain the steady states than an individual population class which perhaps may only happen when the carrying capacity is reached.

We set the proportions as follows: $s(t)=\frac{S[t]}{N(t)}, x(t)=\frac{X(t)}{N(t)}, \quad z(t)=\frac{Z(t)}{N(t)}$, $r(t)=\frac{R(t)}{N(t)}, \quad \nu(t)=\frac{V(t)}{N(t)}$.

Note: $s(t)+\nu(t)+r(t)+x(t)+z(t)=1$

We set $e^{-g a}=\rho$

The equations of $1,2,3,4,5$, with $e^{-g a}=\rho$ become 


$$
\begin{aligned}
\frac{d s(t)}{d t}= & \frac{\rho b_{1} N(t-a)}{N(t)}(1-s(t))-\left(\beta_{1} c_{1} s(t) x(t)+\beta_{2} c_{2} s(t) z(t)\right) \\
& -\alpha s(t)+\eta s(t) x(t)+\lambda s(t) z(t) \\
\frac{d \nu(t)}{d t}= & (\alpha-\delta) s(t)-\left((1-\theta)\left(\beta_{1} c_{1} \nu(t) x(t)+\beta_{2} c_{2} \nu(t) z(t)\right)\right) \\
& -\frac{\rho b_{1} N(t-a)}{N(t) \nu(t)+\eta x(t) \nu[t]+\lambda z(t) \nu(t)} \\
\frac{d r(t)}{d t}= & \delta s(t)-\frac{\rho b_{1} N(t-a)}{N(t)} r(t) \\
& +\eta x(t) r(t)+\lambda r(t) z(t) \\
\frac{d x(t)}{d t}= & \left.\left(\beta_{1} c_{1} x(t) s(t)\right)+\beta_{2} c_{2} s(t) z(t)\right) \\
& +\left((1-\theta)\left(\beta_{1} c_{1} \nu(t) x(t)+\beta_{2} c_{2} \nu(t) z(t)\right)\right) \\
& -(\epsilon+\eta) x(t)-\frac{\rho b_{1} N(t-a)}{N(t)} x(t)+\eta(x(t))^{2}+\lambda x(t) z(t) \\
\frac{d z(t)}{d t}= & \epsilon x(t)-\lambda z(t)-\frac{\rho b_{1} N(t-a)}{N(t)} z(t) \\
& +\eta x(t) z(t)+\lambda(z(t))^{2}
\end{aligned}
$$

In a Disease free system, we have

$$
N(t)=N_{0} e^{(r t)} \Longrightarrow N(t)=N(t-a) e^{(r a)}
$$

With $r=b_{1}-\mu$

We set $w_{1} N(t)=N(t-a)$,

Where $w_{1}$, denotes the weight given to the population size a time (a) earlier. Again we let $s^{*}(t), \nu^{*}(t), r^{*}(t), x^{*}(t), z^{*}(t)$, be the equilibrium points. The equilibrium equations of $7,8,9,10,11$, becomes

$$
\begin{aligned}
0= & \rho b_{1} w_{1}\left(1-s^{*}(t)\right)-\left(\beta_{1} c_{1} s^{*}(t) x^{*}(t)+\beta_{2} c_{2} s^{*}(t) z^{*}(t)\right) \\
& -\alpha s^{*}(t)+\eta s^{*}(t) x^{*}(t)+\lambda s^{*}(t) z^{*}(t) \\
0= & (\alpha-\delta) s^{*}(t)-\left((1-\theta)\left(\beta_{1} c_{1} \nu^{*}(t) x^{*}[t]+\beta_{2} c_{2} \nu^{*}(t) z^{*}(t)\right)\right) \\
& -\rho b_{1} w_{1} \nu^{*}(t)+\eta x^{*}(t) \nu^{*}(t)+\lambda z^{*}(t) \nu^{*}(t) \\
0= & \delta s^{*}(t)-\rho b_{1} w_{1} r^{*}(t)+\eta x^{*}(t) r^{*}(t) \\
& +\lambda r^{*}(t) z^{*}(t) \\
0= & \left.\left(\beta_{1} c_{1} x^{*}(t) s^{*}(t)\right)+\beta_{2} c_{2} s^{*}(t) z^{*}(t)\right) \\
& +\left((1-\theta)\left(\beta_{1} c_{1} \nu^{*}(t) x^{*}(t)+\beta_{2} c_{2} \nu^{*}(t) z^{*}(t)\right)\right) \\
& -(\epsilon+\eta) x^{*}(t)-\rho b_{1} w_{1} x^{*}(t)+\eta\left(x^{*}(t)\right)^{2}+\lambda x^{*}(t) z^{*}(t) \\
0 & \epsilon x^{*}(t)-\lambda z^{*}(t)-\rho b_{1} w_{1} z^{*}(t) \\
& +\eta x^{*}(t) z^{*}(t)+\lambda\left(z^{*}(t)\right)^{2}
\end{aligned}
$$




\subsubsection{Testing the Stability of the Steady state solutions of the D.F.E}

At the disease free equilibrium (D.F.E), we have $\left[s^{*}(t), \nu^{*}(t), r^{*}(t), x^{*}(t), z^{*}(t)\right]=\left[s^{*}(t), \nu^{*}(t), r^{*}(t), 0,0\right]$. Since there is no disease, there is no need to vaccinate thus we use the D.F.E of $[1,0,0,0,0]$, We find the eigenvalues of the Jacobian of the equations

$12,13,14,15,16$, which is given by:-

$$
\left(\begin{array}{ccccc}
-\rho b_{1} w_{1}-\alpha & 0 & 0 & \eta-\beta_{1} c_{1} & \lambda-\beta_{2} c_{2} \\
(\alpha-\delta) & -\rho b_{1} w_{1} & 0 & 0 & 0 \\
\delta & 0 & -\rho b_{1} w_{1} & 0 & 0 \\
0 & 0 & 0 & \beta_{1} c_{1}-\rho b_{1} w_{1}-(\epsilon+\eta) & \beta_{2} c_{2} \\
0 & 0 & 0 & \epsilon & -\lambda-\rho b_{1} w_{1}
\end{array}\right)
$$

The the eigenvalues are given as:

$$
\begin{aligned}
\lambda_{1}= & -\rho b_{1} w_{1} \\
\lambda_{2}= & -\rho b_{1} w_{1} \\
\lambda_{3}= & -\rho b_{1} w_{1}-\alpha \\
\lambda_{4}= & \frac{1}{2}\left(-2 \rho b_{1} w_{1}+c_{1} \beta_{1}-\epsilon-\eta-\lambda\right)-\frac{1}{2} \\
& \left(\sqrt{c_{1}^{2} \beta_{1}^{2}-2 c_{1} \beta_{1} \epsilon+\epsilon^{2}-2 c_{1} \beta_{1} \eta+2 \epsilon \eta+\eta^{2}+2 c_{1} \beta_{1} \lambda-2 \epsilon \lambda-2 \eta \lambda+\lambda^{2}+4 \epsilon \beta_{2} c_{2}}\right) \\
\lambda_{5}= & \frac{1}{2}\left(-2 \rho b_{1} w_{1}+c_{1} \beta_{1}-\epsilon-\eta-\lambda\right)+\frac{1}{2} \\
& \left(\sqrt{c_{1}^{2} \beta_{1}^{2}-2 c_{1} \beta_{1} \epsilon+\epsilon^{2}-2 c_{1} \beta_{1} \eta+2 \epsilon \eta+\eta^{2}+2 c_{1} \beta_{1} \lambda-2 \epsilon \lambda-2 \eta \lambda+\lambda^{2}+4 \epsilon \beta_{2} c_{2}}\right)
\end{aligned}
$$

When $c_{1} \beta_{1}<\left(\epsilon+\eta+\lambda+2 \rho b_{1} w_{1}\right)$, and

$$
\sqrt{c_{1}^{2} \beta_{1}^{2}-2 c_{1} \beta_{1} \epsilon+\epsilon^{2}-2 c_{1} \beta_{1} \eta+2 \epsilon \eta+\eta^{2}+2 c_{1} \beta_{1} \lambda-2 \epsilon \lambda-2 \eta \lambda+\lambda^{2}+4 \epsilon \beta_{2} c_{2}}
$$

is not a real number, then the system is stable.

\subsection{Threshold Quantities}

The Threshold for many epidemiology models is the basic reproduction number $R_{0}$ defined as the average number of secondary infections produced when one infected individual is introduced into a host population where everyone is susceptible [20]. For many deterministic epidemiology models, an infection can get started in a fully susceptible population if and only if $R_{0}>1$. Thus the 
basic Reproduction number $R_{0}$, is often considered as the threshold quantity that determines when an infection can invade and persist in a new host population. The reproduction number could be obtained by inspection if we have only one infective class. If the number of infective classes are two or more, then the technique due to Diekmann [25], 1990, called the next generation matrix is more appropriate.

\subsubsection{The next generation matrix}

We have 2 infected compartments $m=2$, in group II given below.

$$
\begin{aligned}
\frac{d x(t)}{d t}= & \left(\beta_{1} c_{1} x(t) s(t)+\beta_{2} c_{2} s(t) z(t)\right)+\left((1-\theta)\left(\beta_{1} c_{1} \nu(t) x(t)+\beta_{2} c_{2} \nu(t) z(t)\right)\right) \\
& -(\epsilon+\eta) x(t)-\rho b_{1} w_{1} x(t)+\eta(x(t))^{2}+\lambda x(t) z(t) \\
\frac{d z(t)}{d t}= & \epsilon x(t)-(\mu+\lambda) z(t)-\rho b_{1} w_{1} z(t)+\mu z(t)+\eta x(t) z(t)+\lambda(z(t))^{2}
\end{aligned}
$$

$\mathrm{F}$ is given by the matrix:

$$
\left(\begin{array}{c}
\left(\beta_{1} c_{1} x(t) s(t)+\beta_{2} c_{2} s(t) z(t)\right)+\left((1-\theta)\left(\beta_{1} c_{1} \nu(t) x(t)+\beta_{2} c_{2} \nu(t) z(t)\right)\right) \\
0
\end{array}\right)
$$

and

$$
V=V_{i}^{-}-V_{i}^{+}=\left(\begin{array}{c}
(\epsilon+\eta) x(t)+\rho b_{1} w_{1} x(t)-\eta(x(t))^{2}-\lambda x(t) z(t) \\
(\lambda) z(t)+\rho b_{1} w_{1} z(t)-\epsilon x(t)-\eta x(t) z(t)-\lambda(z(t))^{2}
\end{array}\right)
$$

The (D.F.E), point of the system has coordinates $\left[s^{*}(t), \nu^{*}(t), r^{*}(t), x^{*}(t), z^{*}(t)\right]=[1,0,0,0,0]$

The derivatives of $\mathrm{F}$ and $\mathrm{V}$ at $[1,0,0,0]$, are given by:

$$
\begin{gathered}
F=\left(\begin{array}{cc}
\beta_{1} c_{1} & \beta_{2} c_{2} \\
0 & 0
\end{array}\right) \\
V=\left(\begin{array}{cc}
\left(\epsilon+\eta+\rho b_{1} w_{1}\right) & 0 \\
-\epsilon & \left(\lambda+\rho b_{1} w_{1}\right)
\end{array}\right) \\
V^{-1}=\left(\begin{array}{cc}
\left(\frac{1}{\rho b_{1} w_{1}+\epsilon+\eta}\right) & 0 \\
\frac{\epsilon}{\left.\rho b_{1} w_{1}+\epsilon \eta\right)\left(\rho b_{1} w_{1}+\lambda\right)} & \frac{1}{\left(\rho b_{1} w_{1}+\lambda\right)}
\end{array}\right) \\
F * V^{-1}=\left(\begin{array}{cc}
\left(\frac{c_{1} \beta_{1}}{\rho b_{1} w_{1}+\epsilon+\eta}+\frac{\left.\beta_{1} c_{1}\right)}{\left(\rho b_{1} w_{1}+\epsilon+\beta_{1} c_{1}\right.}\right. & 0 \\
0 & 0
\end{array}\right)
\end{gathered}
$$

the eigenvalues of $F * V^{-1}$ is given as 


$$
\begin{aligned}
& \lambda_{1}=0 \\
& \lambda_{2}=\left(\frac{\beta_{1} c_{1}}{\left(\rho b_{1} w_{1}+\epsilon+\eta\right)}+\frac{\epsilon \beta_{2} c_{2}}{\left(\rho b_{1} w_{1}+\epsilon+\eta\right)\left(\rho b_{1} w_{1}+\lambda\right)}\right) \\
& \rho\left(F * V^{-1}\right)=R_{0}=\left(\frac{\beta_{1} c_{1}}{\left(\rho b_{1} w_{1}+\epsilon+\eta\right)}+\frac{\epsilon \beta_{2} c_{2}}{\left(\rho b_{1} w_{1}+\epsilon+\eta\right)\left(\rho b_{1} w_{1}+\lambda\right)}\right)
\end{aligned}
$$

Hence if

$$
\left(\frac{\beta_{1} c_{1}}{\left(\rho b_{1} w_{1}+\epsilon+\eta\right)}+\frac{\epsilon \beta_{2} c_{2}}{\left(\rho b_{1} w_{1}+\epsilon+\eta\right)\left(\rho b_{1} w_{1}+\lambda\right)}\right)>1 .
$$

then the infection will invade and persist in a host population of susceptibles.

\section{Numerical simulations of the Model}

To illustrate some of the analytical results in this paper, numerous numerical simulations of the full model were carried out using the following default parameter values.

$b=0.03664-[33]$. The value of 0.04 used in section 4.1 is adjusted to ensure that the ratio of the adults and those under 15 years is at steady state.

$c_{1}=9.9$ - Calculated from the rate of new infections of 55000 per year obtained from the Kenya National Aids Control Council Report of 2007 and the transmission probability of 0.019 above [11].

$\beta_{1} c_{1}-0.1881$ - the force of infection obtained from [11], and the Kenya National Aids Control Council Report of 2007.

$\delta=0.12$ - Obtained by getting the product of the vaccine efficacy assuming the leaky vaccine and the proportion of the susceptible vaccinated [5].

$\beta_{2} c_{2}=0.03762$ - is the force of infection for those treated and counseled. It is estimated that treatment reduces infectiousness by 50 percent From AIDS science [8]. The combined effects of treatment and counseling (Condom use) is assumed to reduce infectiousness further.

$k=e^{-(b-\mu) * a}$ - The weight given to the population size (a) years ago, calculated from the values of $\mu$ and (a) above.

$\rho=e^{(-g * a)}$ - Is the proportion of uninfected children who survive the developmental stage of $(0-a)$ years, calculated from the values of (a) and (g) above. $g=0.015-[11]$. Estimated to be 1.5 times higher than the natural death rate.

$\mu=0.00973-[33], v=0.3-[18], m=0.2-[18], d=0.4-[18]$.

$\beta_{1}=0.019-[11], \theta=0.3-[5], \eta=0.125-[18], \lambda=0.08-[8], \epsilon=0.44-[12]$, $\alpha=0.4$ - Assumed, $a=15-[11]$.

The initial conditions are obtained from the Kenya demographics profile 2010 


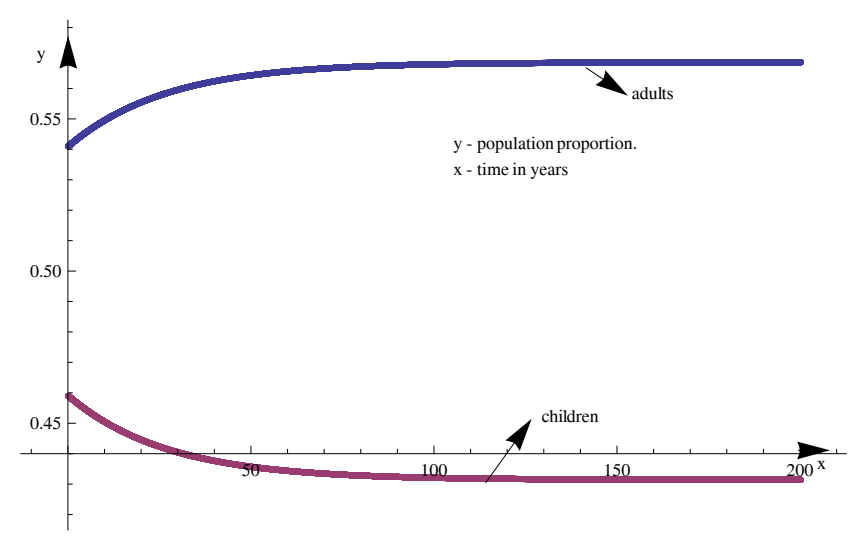

Figure 1: Graph of Population proportions against time in years.

[33], the Kenya National Aids Control Council Report 2007 [32] and the Kenyan population census report of 1999 - 2009 [6].

\subsection{Population model}

To develop a population model when the disease is not incorporated, we set all the disease parameters to zero. We draw the graph of the population proportions with time to investigate wether our parameter values gives us steady state solutions of the population proportions. We need the steady state solutions as a background to study the effects of HIV/AIDS on the stable states. Using the birth rate of 0.03664 , the graph of the population proportions appears as shown in figure 1

The stable solution either requires different initial conditions or different birth and death rates. To obtain a constant population proportion (stable solution) between adults and those under 15 years we adjust the birth rate to 0.04. The new graph is shown in figure 2

\subsection{Treatment of adult infectives with no behavioral change and no change in infectiousness}

We investigate the effects of treatment which does not reduce infectiousness or which is counterbalanced by reckless sexual practices.

The parameter $\epsilon$ is the proportion of infectives who receive treatment. Different parameter values for treatment are simulated and the results are shown in figure 3 . 


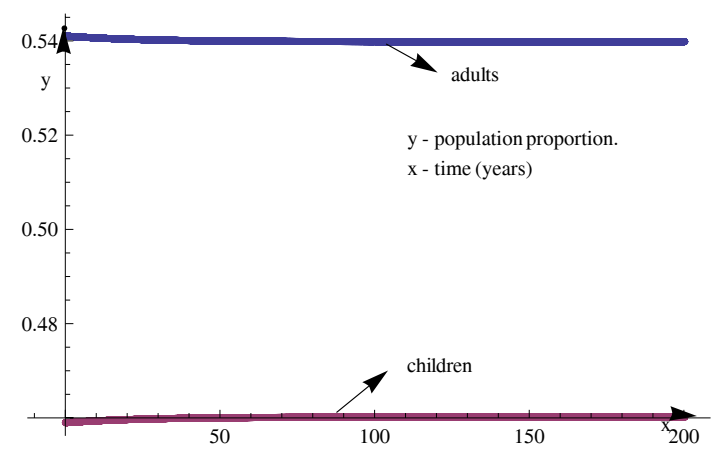

Figure 2: Graph of Population proportions against time in years.

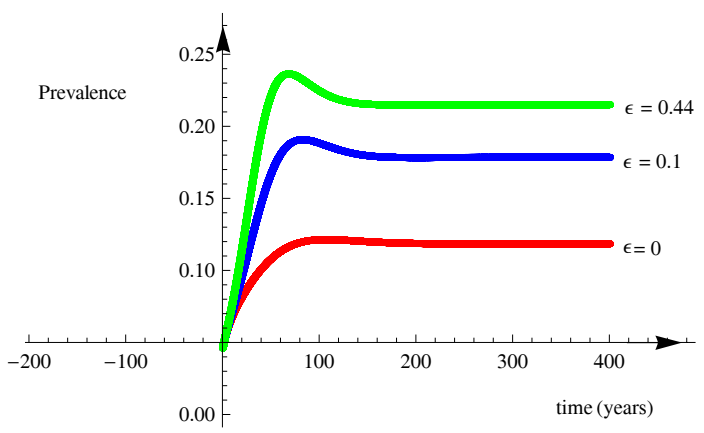

Figure 3: Graph of the disease prevalence against time. When $\epsilon=0, R_{0}=$ 1.39613. When $\epsilon=0.1, R_{0}=1.69441$. When $\epsilon=0.44, R_{0}=1.93215$.

The increase in the disease prevalence level with treatment may be due to the fact that treatment lengthens the lives of the infectives and as such those who would have died of AIDS do become healthier and continue to spread the disease if they are not counseled or the counseling has no effect in changing there social behaviour.

The population proportions of those below 15 years, adults and HIV/AIDS victims are shown in figure 4.

This graph reflects the case when $R_{0}$ exceeds unity with convergence to the disease endemic equilibrium.

\subsection{Treatment of adult infectives with behavioral change and change in infectiousness}

This reflects the current state of HIV/AIDS in Kenya where 44 percent of $\mathrm{HIV} / \mathrm{AIDS}$ infectives receive treatment and counseling. According to medical research done in the U.S.A [10] treatment alone reduces infectiousness by 50 


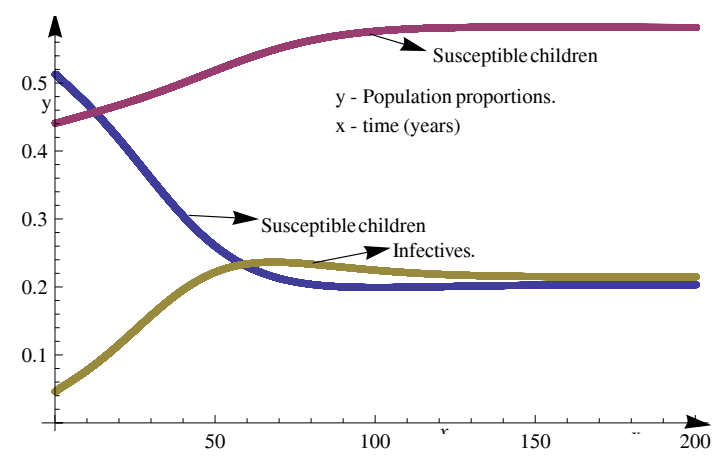

Figure 4: Graph of the population proportions against time, $R_{0}=1.93215$.

percent. In Kenya, no data exist to estimate by which percentage treatment coupled with counseling (zero grazing,condom use and circumcision) reduces infectiousness.

We simulate the prevalence of the disease with time for $\beta_{2} c_{2}=0.0905$ (reducing infectiousness by 50 percent), $\beta_{2} c_{2}=0.07524$ (reducing infectiousness by 60 percent), $\beta_{2} c_{2}=0.03762$ (reducing infectiousness by 80 percent) and $\beta_{2} c_{2}=0.01881$ (reducing infectiousness by 90 percent) as shown in figure 5 .

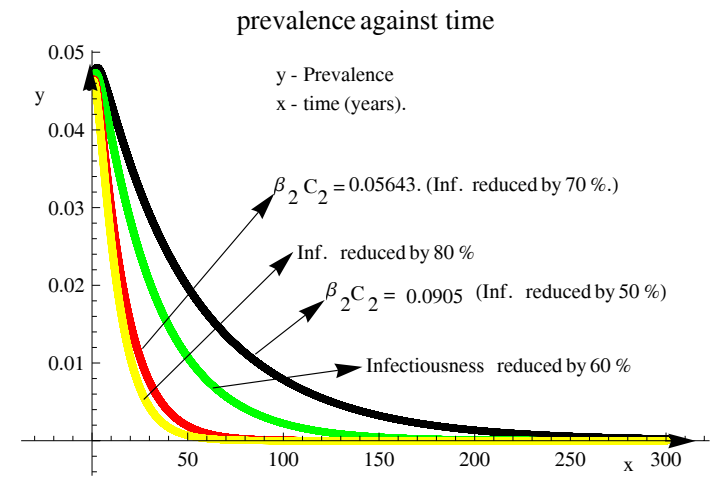

Figure 5: Graph of prevalence against time in years.

We observe that at the estimated current force of infection of $\beta_{1} c_{1}=0.1881$, coupled with treatment alone that reduces infectiousness by 50 percent, the disease will die out in about 200 years time. We thus conclude that counseling and treatment that reduces infectiousness is very effective in controlling the spread of the disease.

Using the force of infection of $\beta_{1} c_{1}=0.1881$, coupled with treatment and counseling that reduces infectiousness by 80 percent $\left(\beta_{2} c_{2}=0.03762\right)$, the disease will always die out even with different initial conditions as shown in figure 6 


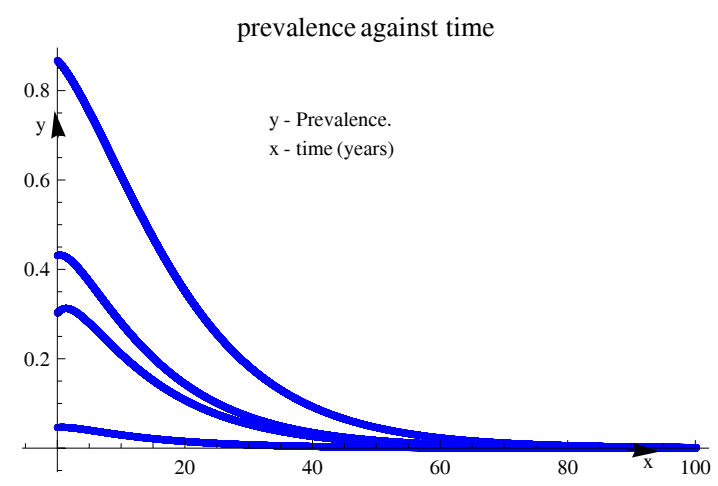

Figure 6: $R_{0}=0.648258$.

This shows convergence to the disease free equilibrium with $R_{0}$ less than unity.

\subsection{Speculative studies}

This area is based on speculative studies in the event that an effective HIV/AIDS vaccine is found which currently is not there though the latest findings in Thailand [5] indicated that some vaccine for some strain of the HIV/AIDS virus has been obtained with an efficacy level of 30 percent. It is not known however that the vaccine acts as the "Leaky type" or as the "All or Nothing type" or both. We assume here that 40 percent of the susceptible are vaccinated and the vaccine acts both as the "leaky" type and the "All or Nothing" type of Vaccine. We begin by investigating the effects of treatment alone without vaccination then simulate for vaccination alone without treatment in a single graph to determine which of them would be more effective assuming that treatment alone reduces infectiousness by 50 percent, i.e $\beta_{2} c_{2}=0.09405$. The simulations are shown in figure 7.

This model suggests that vaccination alone without treatment is more effective in controlling HIV spread using the vaccination and treatment parameters above.

\subsubsection{The trade - off between vaccination and treatment}

In the second step we simulate vaccination alone, treatment alone and a combination of the two within a single graph to determine wether a trade off exists between vaccination and treatment. In this case we again assume that treatment reduces infectiousness by 50 percent as shown in figure 8 . 


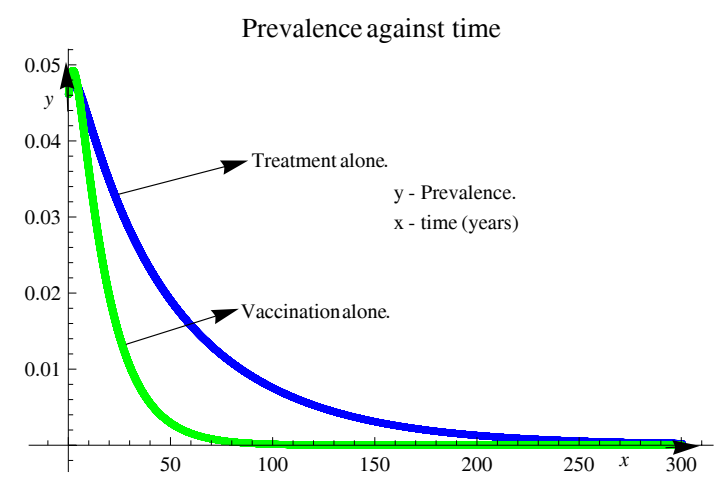

Figure 7: Graph of prevalence against time

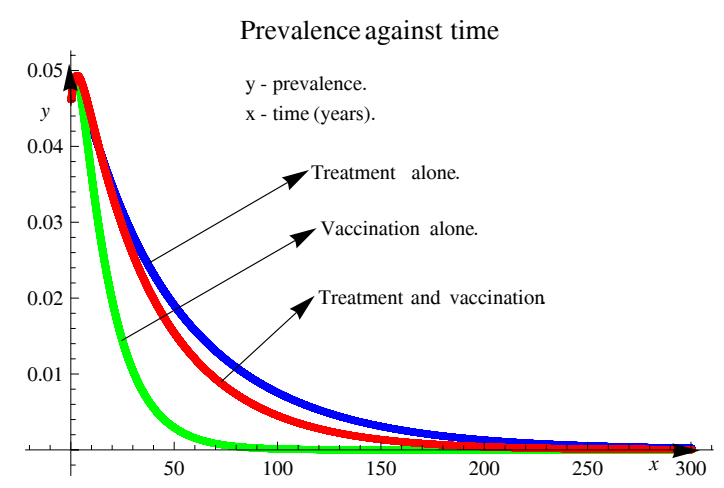

Figure 8: Graph of prevalence against time

We note that a trade - off seems to exist between vaccination and treatment using our parameter values for treatment and vaccination. We observe that vaccination alone is still more effective than a combination of treatment and vaccination implying that treatment would be counter productive when applying vaccination.

\subsubsection{Threshold Parameters}

We set to numerically investigate the thresholds of the disease transmission rates beyond which treatment and vaccination is counterproductive by incorporating counseling in treatment. We assume here that counseling and treatment reduces infectiousness by 70 percent i.e $\beta_{2} c_{2}=0.05643$. The graph appears as shown in figure 9.

We observe that treatment and counseling that reduces infectiousness by 70 percent coupled together with vaccination is equivalent to applying vaccination alone thus Counseling coupled with treatment that reduces infectiousness by 


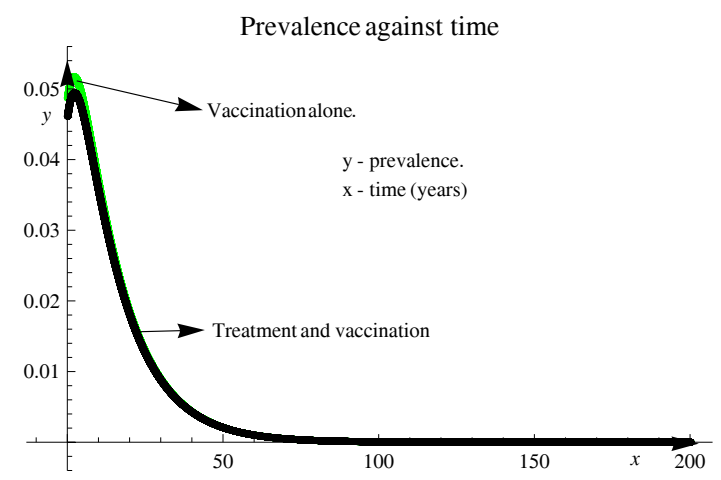

Figure 9: Graph of prevalence against time

less than 70 percent will be counterproductive if applied together with vaccination whereas if infectiousness is reduced by more than 70 percent as a result of treatment and counseling then a combination of treatment,counseling and vaccination would not be counterproductive using our parameters above.

Assuming that treatment coupled with counseling reduces infectiousness by 80 percent, we set to simulate the effects of the same in terms of prevalence. The simulations for the prevalence appears as shown in figure 10.

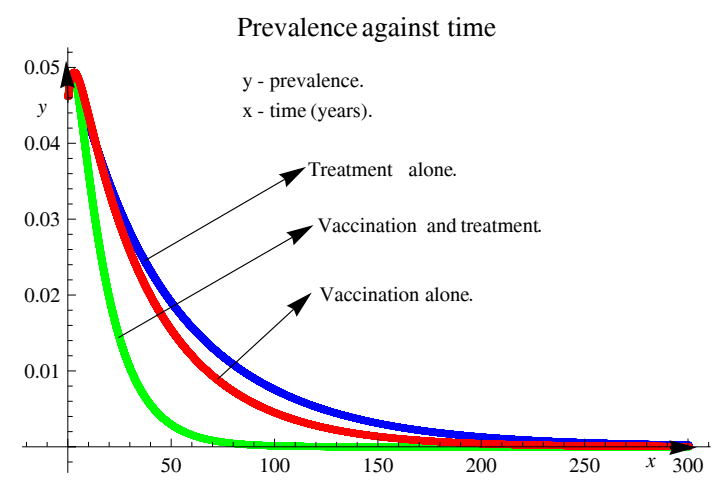

Figure 10: Graph of prevalence against time

It is important to note here that a combination of treatment, counseling and vaccination would be more effective than each one of them applied separately.

\section{Conclusion and Recommendations}

We formulated a comprehensive HIV/AIDS transmission model with reference to the Kenyan situation and according to this model, we observed the following: 
- If there is no intervention even at the current reduced rates of HIV/AIDS infection, our model predicts that we might get a lower proportion of adults in the population than those who are 15 years and below which would mean that we have more dependants than the workforce in the country.

- Treatment that does not reduce infectiousness is worse than when the treatment is not applied at all, however when coupled with effective counseling, then it is very effective in combating the spread of the disease and finally eliminating it.

- Our speculative studies for vaccination showed that careful considerations should be made when a combination of vaccination and treatment is to be applied because a combination of the two could be counterproductive or helpful depending on how it is implemented.

This study provides an in-depth mathematical analysis of a practical comprehensive HIV/AIDS transmission model for the transmission dynamics of HIV/AIDS with special reference to Kenya. It should be noted however that deterministic Mathematical modeling is more useful in predicting trends of diseases usually over longer periods of time but might not be very accurate in the short run because of changing circumstances.

\section{Acknowledgments}

We would like to thanked Dr. David Stern of Maseno University for his useful comments.

\section{References}

[1] A.G. Mckendric, Applications of mathematics to medical problems, Proc. Edinburgh Math. Soc.,44 (1926).

[2] A.M, Johnson, R. M. Anderson, G.P. Medley and R.M. May, A preliminary study of the transmission dynamics of the Human Immunodeficiency virus, the causative agent of AIDS, J. Math Appl. Med. Biol., 3 (1986), pp. $229-263$.

[3] A.R Kimbir and H.K. Oduwole, A mathematical model of HIV/AIDS: Transmission dynamics considering counseling and antiretroviral therapy. Medwell Journal of Modern Mathematics and Statistics. 2(5): 166 169,2008 .

[4] A.W. Wells, Even imperfect vaccines could be valuable. 1998. 
[5] BBC News: Available online at news.bbc.co.uk/2/hi/8272113.stm. $3 / 02 / 2010$.

[6] Business Daily, Kenya. 6th, september, 2010.

[7] Bureau of Hygiene and Tropical Diseases 'AIDS Newsletter', 13 (2) p.13 CDC (1998) 'Short course regimen of AZT proven effective in reducing perinatal HIV transmission: offers hope for reducing mother-to child HIV transmission in developing world', Press release, February 18.(1998).

[8] Calculating the potential epidemic - Level impact of therapeutic vaccination on the San Francisco HIV epidemic. AIDS SCIENCE. Volume 3 No. 21,2003. Available online at http://aidsscience.org/Articles/AIDSSCIENCE040.asp on 22/04/2010.

[9] D. Bernoulli, Essai d'une. nouvelle analyse de la mortalite causee par la petite verole et des avantages de l'inoculation pour la prevenir, in memoires de mathematiques et de physique, Academie Royale des sciences, Paris, 1760 .

[10] Efficacy of Anti- Retroviral drugs in reducing M.T.C.T of HIV in Africa A media- Analysis of published chemical trials. Available online at http://www.ncbi.n/m.nih.gov/pubmed/18544018. 22/04/2010.

[11] F. Baryama and J.Y.T. Mugisha, Comparison of single - stage and staged progression models for HIV/AIDS models, International Journal of Mathematics and Mathematical sciences. Volume 2007, Article I.D. 18908, November 2007.

[12] HIV and AIDS in Kenya. Available online at http://www.avert.org/hivkenya.htm.. 27/04/2010.

[13] HIV/AIDS Policy Fact Sheet: The HIV/AIDS Epidemic in Kenya, October 2009, Available online at www.kff.org

[14] H.Y. Hsieh, A two sex model for treatment of AIDS and behaviour change in a population of varying size, IMA. J. Applied Bio. Biosci., 167: 1 - 18. 1996.

[15] H.W. Hethcote, Modeling AIDS prevention programs in a population of homosexual in modeling the AIDS epidemic: Planing, Policy and Prediction, eds, Raven press, New York, 1994, pp 91 - 107.

[16] H. Velasco and Y. Hsieh, Modeling the effect of treatment and behaviour change in HIV transmission dynamics, J. Math. Biol.,32. 1994. 
[17] H. Ying - Yen and K. Cooke, Behaviour change and treatment of core groups: it's effects on the spread of HIV/AIDS. IMA J. Math. App. Med. Biol.,17, 2000.

[18] J.Y.T. Mugisha and L.S. Luboobi, Treatments of HIV/ AIDS Patients: A mathematical perspective. International Journal of Management and Systems. 16(2): 220 - 243. 1999.

[19] J.Y.T. Mugisha and L.S. Luboobi, Modeling the effect of vertical transmission in the dynamics of HIV/AIDS in an age-structured population, International Journal of management and Systems, 2003.

[20] L. Mathew, G. Andrew, V.V. Paul and K. Susan, Modeling the effects of combination antiretroviral treatments on H.I. V incidence, National Centre in HIV epidemiology an clinical Research, University of New South Wales, Darlinghurst, Australia. 2001.

[21] L. Odeny, HIV/AIDS Vaccine Model with application to kenya, M.S.C thesis Maseno University College Kenya. 2003.

[22] M. Kgosimore and E.M. Lungu, Effects of vaccination and treatment on the Spread of HIV/AIDS, J. Biol. Syst. 12(4):399 - 417, 2006.

[23] M.A, Umar O.E. Olowofeso and R.A. Ademiluyi, Modeling horizontal transmission of HIV/AIDS in an age structured population, Medwell Journal of Modern mathematics and Statistics 2(6) :174 - 180.2008

[24] N.F. Britton, Essential Mathematical Biology, sringer, Berlin. pp. 83 108. 1990.

[25] O. Diekmann, J.A.P. Heesterbeek and J.A.J. Metz, The defination and the computation of the basic reproductive ratio $R_{0}$ in models for infectious diseases in heterogeneous populations, J. Math. Biol., 28 (1990). pp. 365 - 382

[26] R.M. Anderson and R.M. May, Infectious diseases of humans: Dynamics and control, Oxford University Press, Oxford U.K., 1991.

[27] R.O. Simwa and G.P. Pokhariyal, A dynamic model for stage specific $H I V / A I D S$ incidences with application to sub- saharan Africa, Elsevier Science. 2002.

[28] S.R. Arni and R. Srinivasa, Mathematical modeling of AIDS epidemic in India, Centre for Ecological Sciences. Current Science VOL.84, NO 9, 10 MAY 2003. 
[29] S.M. Blower, K. Koelle, D.E. Kirsscher and J. Mills, Live attenuated HIV/AIDS Vaccines: Predicting the trade- off between efficacy and safety. PNAS 98(6):368 - 3623. 2001.

[30] S.P. Blynthe and R.M. Anderson, Heterogeneous sexual activity models of HIV transmision in male homosexual populations, J. Math. Appl. Med. Biol. 5(4). 1988.

[31] T. M. Amunga, HIV/AIDS modeling: Leaky type of Vaccine, M.S.C thesis Maseno University, Kenya. 2008.

[32] Kenya National AIDS Control Council Report, 2007, Available online at http://www.kaisernetwork.org.

[33] The Kenya Demographics profile 2010, Available online at http :

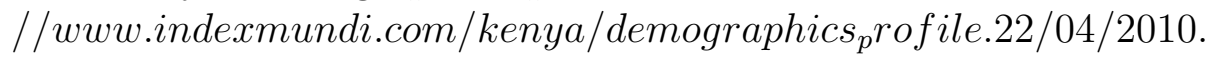

[34] UNAIDS/WHO, Uganda:Epidemiological fact sheet on HIV/AIDS and sexually transmitted infections - 2002 update.

[35] UNAIDS, "Africa fact sheet", march 2000.

[36] W.H. Hamer, Epidemic disease in England, Lancet, 1. 1906.

[37] W.O. Kermack and A.G. Mckendric, Contributions to the mathematical theory of epidemics, part 1, Proc. R. Sec. London, 1927, A115, 700 - 721.

[38] Workshop on HIV/AIDS and Adult Mortality in Developing Countries: Population Division, Department of Economic and Social Affairs United Nations Secretariat, New York, 8-13, September 2003.

\section{Received: June 21, 2012}

